\title{
Incidence of myocardial injury after non-cardiac surgery: Experience at Groote Schuur Hospital, Cape Town, South Africa
}

\author{
E Coetzee, ${ }^{1}$ MB ChB, DA (SA), FCA (SA); B M Biccard, ${ }^{1}$ MB ChB, FFARCSI, FCA (SA), MMed (Anaesth), PhD; \\ R A Dyer, ${ }^{1}$ BSc Hons, MB ChB, FCA (SA), PhD; N D Meyersfeld, ${ }^{2} \mathrm{MB}$ ChB, FCA (SA); C Chishala, ${ }^{3}$ MB ChB, FCP (SA); \\ B M Mayosi, ${ }^{4,5}$ BMedSci, MB ChB, FCP (SA), DPhil, AMP \\ ${ }^{1}$ Department of Anaesthesia and Perioperative Medicine, Groote Schuur Hospital and Faculty of Health Sciences, University of Cape Town, \\ South Africa \\ ${ }^{2}$ Private practice, Southern Anaesthetic Associates, Cape Town, South Africa \\ ${ }^{3}$ Department of Cardiology, Groote Schuur Hospital and Faculty of Health Sciences, University of Cape Town, South Africa \\ ${ }^{4}$ Dean's Office, Faculty of Health Sciences, University of Cape Town, South Africa \\ ${ }^{5}$ Department of Medicine, Groote Schuur Hospital and Faculty of Health Sciences, University of Cape Town, South Africa
}

Corresponding author: E Coetzee (ettiennec@gmail.com)

\begin{abstract}
Background. Myocardial injury after non-cardiac surgery (MINS) is a newly recognised entity identified as an independent risk factor associated with increased 30-day all-cause mortality. MINS increases the risk of death in the perioperative period by $\sim 10$-fold. More than $80 \%$ of patients with MINS are asymptomatic, so the majority of diagnoses are missed. Awareness of MINS is therefore important for perioperative physicians.

Objectives. To investigate the incidence of MINS after elective elevated-risk non-cardiac surgery at Groote Schuur Hospital, Cape Town, South Africa (SA).

Methods. Patients aged $\geq 45$ years undergoing elective elevated-risk non-cardiac surgery were enrolled via convenience sampling. The new fifth-generation high-sensitivity cardiac troponin T blood test was used postoperatively to identify MINS. Preoperative troponin levels were not measured.

Results. Among 244 patients included in the study, the incidence of MINS was 4.9\% (95\% confidence interval (CI) 2.8 - 8.5), which was not significantly different from that in a major international prospective observational study (VISION) $(8.0 \%$ ( $95 \%$ CI $7.5-8.4)$ ); $p=0.080$ Conclusions. Our SA cohort had a lower cardiovascular risk profile but a similar incidence of MINS to that described in international literature. The impact of MINS on morbidity and mortality is therefore likely to be proportionally higher in SA than in published international studies. The limited sample size and lower event rate weaken our conclusions. Larger studies are required to establish patient and surgical risk factors for MINS, allowing for revision of cardiovascular risk prediction models in SA.
\end{abstract}

S Afr Med J 2018;108(5):408-412. DOI:10.7196/SAMJ.2018.v108i5.12784

Recent reports estimate global annual perioperative morbidity and mortality rates to be $\sim 3 \%$ and $0.5 \%$, respectively. ${ }^{[1]}$ One-third of deaths in patients undergoing non-cardiac surgery are cardiovascular in origin. ${ }^{[2]}$ Myocardial ischaemia with subsequent injury is strongly associated with perioperative mortality. ${ }^{[3,4]}$ A large multicentre international study in 2012 investigated the mortality associated with perioperative elevated troponin levels in $>15000$ patients from North and South America, Australia, Europe and Asia (Vascular Events In Non-cardiac Surgery Patients Cohort Evaluation (VISION) study). ${ }^{[3]}$ The 30-day mortality was found to be independently associated with myocardial injury after non-cardiac surgery (MINS) in a subsequent analysis of the data. ${ }^{[5]}$ Ten percent of patients with myocardial injury died within 30 days of surgery. ${ }^{[5]}$ Eighty-four percent of perioperative patients with myocardial injury were asymptomatic, resulting in missed diagnosis in the vast majority of patients with MINS. ${ }^{[5]}$ Thirty-day mortality was found to be independently associated with an elevated cardiac biomarker, specifically cardiac troponin $\mathrm{T}$ (cTnT).

The VISION study utilised the previous fourth-generation cTnT test. ${ }^{[3]}$ MINS has been defined as an isolated fourth-generation cTnT level of $\geq 30 \mathrm{ng} / \mathrm{L}(0.03 \mu \mathrm{g} / \mathrm{mL})$ due to myocardial ischaemia, which predicted mortality with an odds ratio of 10.07 (95\% confidence interval (CI) 7.84 - 12.94). ${ }^{[5]}$ A substudy of the VISION investigation suggested that a suitable cut-off level for the more sensitive fifthgeneration cTnT (hs-cTnT) for a diagnosis of MINS is $>33 \mathrm{ng} / \mathrm{L} .^{[6]}$

With regard to MINS, only a single vascular surgical cohort has been studied in South Africa (SA). ${ }^{[7-9]}$ Because of differing patient characteristics and risk profiles, extrapolation of the incidence of MINS from international data to SA surgical patients may be inappropriate.

\section{Objectives}

The primary objective of this study was to determine the incidence of MINS after non-cardiac surgery in an elective elevated-risk surgical population in SA. The null hypothesis for this study was that the incidence of MINS would be similar in the SA study population to that described in peer-reviewed international reports. Secondary outcomes were the prevalence of risk factors such as patient demographics, surgical categories and comorbidities associated with MINS.

\section{Methods}

This was a prospective, single-centre observational study investigating the incidence of MINS at Groote Schuur Hospital, a tertiary referral 
centre in Cape Town, SA. Patients aged $\geq 45$ years presenting for elective elevated-risk non-cardiac surgery were eligible. Elevatedrisk surgery was defined as all surgery with a predicted risk of major adverse cardiac events of $>1 \% .{ }^{[10]}$ This included all intra-abdominal, non-cardiac thoracic, joint replacement, major orthopaedic and vascular surgery. Patients were selected by means of convenience sampling from November 2014 to February 2016, and only these patients were included in the study.

Enrolled patients were investigated for MINS, defined as an elevated postoperative hs-cTnT level suspected to be due to myocardial ischaemia, with no other known non-ischaemic causes of troponin elevation. Once patients were enrolled, postoperative blood samples were collected. Specimens were analysed using the Troponin $\mathrm{T}$ hs (highly sensitive) immunoassay (Roche Diagnostics, Germany).

After approval from the Human Research Ethics Committee of the University of Cape Town (ref. no. 818/2014), data collection was commenced. Theatre lists were interrogated and patients with appropriate inclusion criteria were identified. Informed consent was obtained postoperatively, after which appropriate blood samples were taken. In the majority of patients (90.6\%), one blood sample was taken within 24 hours of surgery. In the remainder, a second sample was taken within 72 hours. If two samples were taken, the highest troponin elevation was recorded. MINS was diagnosed if the postoperative hs-cTnT level was $>33 \mathrm{ng} / \mathrm{L}$.

As sampling only commenced after surgery, we could not identify patients with pre-existing cTnT elevation. Known preoperative myocardial infarction, pulmonary embolism, sepsis and renal dysfunction were therefore also grounds for exclusion because of the direct or indirect influence of these conditions on measured postoperative cTnT. Renal impairment was defined using international guidelines. ${ }^{[11,12]}$ Further exclusion criteria included pre-existing hypotension and low-risk surgery such as ophthalmic and superficial plastic surgery. Other comorbidities were identified during the patient interview. Postoperative mortality data were not collected.

A post hoc decision was made to present some of the key characteristics of the Groote Schuur Hospital cohort, the SA vascular surgery cohort and the VISION cohort in order to provide further insight into the incidence of MINS in the Groote Schuur patients. ${ }^{[3,8]}$ A statistical comparison of these characteristics between the Groote Schuur cohort and the VISION cohort was conducted.

\section{Statistical analysis}

Based on previous studies, a prevalence of MINS of 10 - 15\% was estimated for our study population. On this basis, a sample size of 219 - 266 would be required for a $95 \%$ CI of $10 \%$ for the estimate of the prevalence of MINS. A sample size of 300 was targeted.

Statistical analysis was done using Minitab 17 Statistical Software (Minitab, USA). ${ }^{[13]}$ Continuous variables were described using sample means and standard deviations (SDs) and categorical variables using sample medians and interquartile ranges. Where statistical comparisons were made between continuous variables, the unpaired $t$-test was used, while categorical data were compared using the $\chi^{2}$ test or the $\chi^{2}$ test with Yates's correction, as appropriate.

\section{Results}

During the data collection period (16 months), theatre lists were actively interrogated for only 39 weeks owing to researcher availability. For this reason, convenience sampling was our method of selection. During that time, $\sim 1100$ patients underwent elective surgery, of whom 301 were eligible. The flow diagram of patient recruitment is shown in Fig. 1. The majority of initial exclusions were because of inappropriate age and/or low-risk surgery. The final number of patients included was 244 .

The patient demographic details, surgical categories and comorbidities are shown in Table 1. The majority of patients were in the younger age category, 45 - 65 years. General and gynaecological

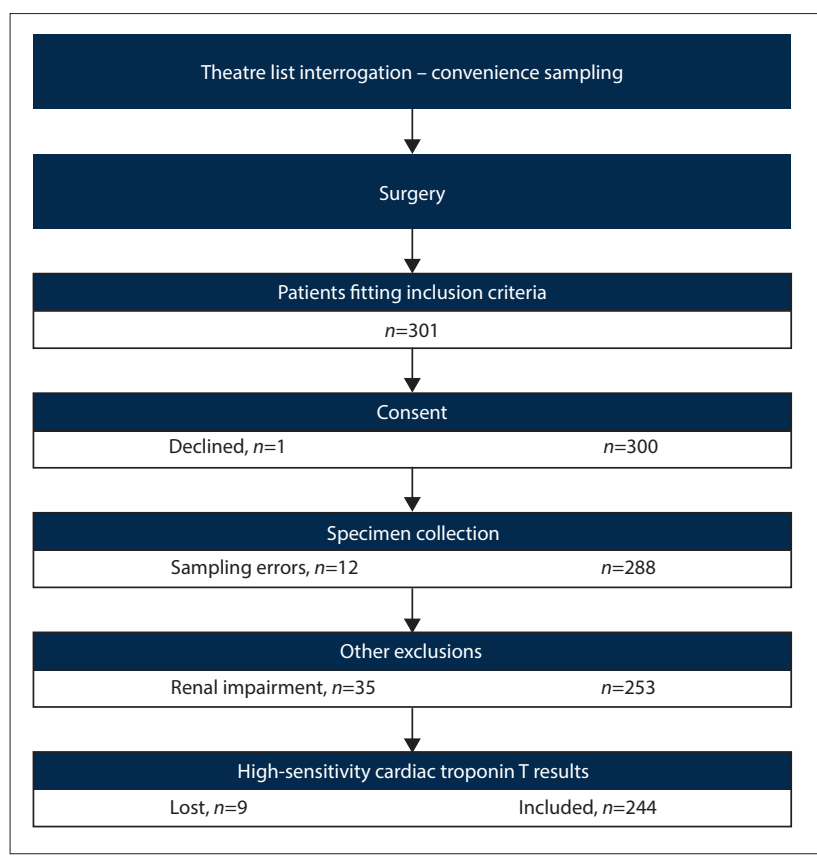

Fig. 1. Flow diagram of patient recruitment.

\begin{tabular}{|c|c|}
\hline Included patients, $N$ & 244 \\
\hline Females, $n(\%)$ & $139(57.0)$ \\
\hline \multicolumn{2}{|l|}{ Age (years) } \\
\hline Mean (SD) & $60.5(9.4)$ \\
\hline $45-64, \%(95 \% \mathrm{CI})$ & $68.9(62.8-74.3)$ \\
\hline $65-75, \%(95 \% \mathrm{CI})$ & $23.8(18.8-29.5)$ \\
\hline$>75, \%(95 \% \mathrm{CI})$ & $6.6(4.0-10.5)$ \\
\hline \multicolumn{2}{|l|}{ Surgical categories, $n(\%)$} \\
\hline General & $63(25.8)$ \\
\hline Gynaecological & $52(21.3)$ \\
\hline Orthopaedic & $40(16.4)$ \\
\hline Urological & $27(11.1)$ \\
\hline Vascular & $18(7.4)$ \\
\hline Otolaryngological & $16(6.6)$ \\
\hline Thoracic & $9(3.7)$ \\
\hline Neurological & $3(1.2)$ \\
\hline \multicolumn{2}{|l|}{ Comorbidities ${ }^{*}$} \\
\hline Median (IQR) & $1(1-2)$ \\
\hline Hypertension, $n(\%)$ & $161(66.0)$ \\
\hline Malignancy, $n(\%)$ & $78(32.0)$ \\
\hline Smoking, $n(\%)$ & $69(28.3)$ \\
\hline Diabetes mellitus, $n(\%)$ & $50(20.5)$ \\
\hline Hypercholesterolaemia, $n(\%)$ & $47(19.3)$ \\
\hline Previous myocardial infarction, $n(\%)$ & $20(8.2)$ \\
\hline Previous stroke, $n(\%)$ & $15(6.1)$ \\
\hline Previous heart failure, $n(\%)$ & $6(2.5)$ \\
\hline
\end{tabular}


surgery were the most common operations performed, which explains the higher proportion of female participants. Hypertension and malignancy were the most frequently recorded comorbidities. The hs-cTnT results are reported in Table 2.

The incidence of MINS was $4.9 \%$ (95\% CI 2.8 - 8.5). There were inadequate data for multivariate regression analysis to investigate associations between patient or surgical risk factors and MINS.

Comparisons between the Groote Schuur, SA vascular and VISION cohorts are shown in Table 3. There was no significant difference in the incidence of MINS between the Groote Schuur and VISION studies. The Groote Schuur cohort had younger patients, fewer male patients and more hypertension. The Groote Schuur patients underwent more elective and vascular surgery than the VISION cohort.

\section{Discussion}

The main finding of this study was that the incidence of MINS in the elective elevated-risk surgical population in SA was comparable to reports from a large international study. It was impossible to perform a risk-adjusted analysis to compare the incidence of MINS between the Groote Schuur and VISION cohorts. A previous comparison between the South African Surgical Outcomes Study (SASOS) and the European Surgical Outcomes Study (EuSOS) suggested that SA surgical patients have a lower risk profile than surgical patients in Europe. ${ }^{[14]}$ The patients in our study probably had a lower cardiovascular risk profile, as they were younger, included fewer males and had more elective surgery than the VISION cohort, as seen in Table 3. This has important public health implications for SA, because of the major morbidity and mortality associated with MINS.

Current perioperative morbidity rates translate into major complications in $\sim 7$ million patients and 1 million deaths per

\begin{tabular}{|c|c|}
\hline cTnT undetectable, $n(\%)$ & $42(17.2)$ \\
\hline cTnT (ng/L), median (IQR) & $7(4-14)$ \\
\hline cTnT (ng/L), median >33 ng/L (IQR) & $60(37-83)$ \\
\hline cTnT >99th percentile*, $n(\%)(95 \%$ CI $)$ & $55(22.5)(17.8-28.2)$ \\
\hline cTnT >33 ng/L $\mathrm{L}^{\dagger}, n(\%)(95 \% \mathrm{CI})$ & $12(4.9)(2.8-8.5)$ \\
\hline cTnT > 53 ng/L, $n(\%)(95 \%$ CI) & $7(2.9)(1.3-5.9)$ \\
\hline
\end{tabular}

annum in the immediate perioperative period, since $>200$ million surgical operations are performed worldwide per year. ${ }^{[1]}$ Studies from high-income countries suggest that $50 \%$ of major adverse perioperative events could be avoided. ${ }^{[1]}$ Research on the cause and prevention of perioperative complications could therefore benefit global healthcare.

Myocardial ischaemia remains a major cause of perioperative morbidity and mortality. ${ }^{[15]}$ To provide some perspective, $7 \%$ of medical patients who are treated in hospital for an acute myocardial infarction die within 30 days. ${ }^{[16]}$ Patients may present with myocardial ischaemia either as a medical emergency or in the perioperative period. Both appear to have a significant 30-day mortality, but their clinical presentations are very different. The diagnosis of myocardial infarction in medical patients relies on clinical symptoms and signs in addition to positive special investigations (electrocardiogram, cardiac biomarkers, cardiac imaging studies). ${ }^{[17]}$ A recent review suggests that the high proportion of silent presentations and the lack of high-grade evidence for therapeutic interventions could explain the hesitancy of perioperative clinicians to adopt MINS as a significant perioperative event. ${ }^{[18]}$ However, it is clear that MINS results in significant morbidity and mortality and poses a significant public health burden. ${ }^{[5]}$

Cardiac biomarkers utilised for the diagnosis of myocardial infarction require predefined reference limits. These limits or cutoff values are derived from healthy individuals. ${ }^{[19-21]}$ Analysis of data from the VISION study showed not only that elevation of the cardiac biomarker cTnT was the strongest predictor of 30-day mortality, but also that the magnitude of troponin elevation correlated with risk. The VISION study attempted to predict mortality related to increasing levels of fourth-generation cTnT, in combination with the clinical presentation. Adding positive symptomatology to the model did not change the diagnostic threshold, and the diagnosis had the same prognostic importance for mortality as a myocardial infarction diagnosed using the Universal Definition of Myocardial Infarction. ${ }^{[5]}$

Correlations between the fourth- and fifth-generation cTnT test do exist. A fourth-generation cTnT level of $30 \mathrm{ng} / \mathrm{L}$ correlates with a fifth-generation cTnT (hs-cTnT) level of $\sim 53 \mathrm{ng} / \mathrm{L}$. However, a substudy of the VISION investigation found that the 95 th percentile value for perioperative patients was $33 \mathrm{ng} / \mathrm{L}$ (95\% CI 26 - $49 \mathrm{ng} / \mathrm{L}$ ) for hs-cTnT. ${ }^{[6,22]}$ The cumulative proportion of patients with a fourthgeneration cTnT level $>30 \mathrm{ng} / \mathrm{L}$ was $9 \%$, compared with $7-10 \%$ $>33 \mathrm{ng} / \mathrm{L}$ using the hs-cTnT.

Table 3. Comparison between the GSH cohort, the SA vascular surgical cohort and the VISION study ${ }^{[3,8]}$

\begin{tabular}{|c|c|c|c|c|}
\hline & GSH $(N=244)$ & $\begin{array}{l}\text { SA vascular c } \\
(N=788)\end{array}$ & VISION $(N=15133)$ & $p$-value ${ }^{*}$ \\
\hline Age (years), mean (SD) & $60.5(9.4)$ & $58.3(14.2)$ & $\mathrm{NR}$ & \\
\hline Age 45 - 64 years, $n(\%)$ & $168(68.9)$ & NR & $7436(50.9)$ & $<0.001$ \\
\hline Males, $n(\%)$ & $105(43.0)$ & $512(65.0)$ & $7339(49.5)$ & 0.044 \\
\hline Hypertension, $n(\%)$ & $161(66.0)$ & $540(68.5)$ & 7709 (50.9) & $<0.001$ \\
\hline Diabetes mellitus, $n$ (\%) & $50(20.5)$ & $338(42.9)$ & $2952(19.5)$ & 0.761 \\
\hline History of coronary artery disease, $n(\%)$ & $20(8.2)$ & $275(34.9)$ & $1832(12.1)$ & 0.078 \\
\hline History of stroke, $n(\%)$ & $15(6.1)$ & $159(20.2)$ & $696(4.6)$ & 0.323 \\
\hline History of heart failure, $n$ (\%) & $6(2.5)$ & $37(4.7)$ & $703(4.6)$ & 0.151 \\
\hline Vascular surgery, $n(\%)$ & $18(7.4)$ & $788(100)$ & $504(3.3)$ & 0.001 \\
\hline Elective surgery, $n(\%)$ & $244(100)$ & NR & $12991(85.8)$ & $<0.001$ \\
\hline Incidence of MINS, $n / N(\%)$ & $12 / 244(4.9)$ & $25 / 509(5.0)^{\dagger}$ & $1200 / 15065(8.0)$ & 0.080 \\
\hline
\end{tabular}


The fifth-generation cTnT (hs-cTnT) used in the present study has improved diagnostic sensitivity to nearly $100 \%$ in patients presenting as a medical emergency; a level $>14 \mathrm{ng} / \mathrm{L}$ suggests myocardial necrosis. ${ }^{[21,23]}$ The increased sensitivity has added complexity to the interpretation of perioperative changes in troponin levels, and specificity is reduced because of the many alternative causes of troponin elevation during this period. ${ }^{[21]}$ Carbon monoxide poisoning, renal failure, heart failure, acute pericarditis, acute exacerbation of chronic obstructive airway disease, chemotherapyrelated myocardial damage, pulmonary hypertension, pulmonary embolism, sepsis, stroke and subarachnoid haemorrhage can all result in cTnT elevations. ${ }^{[24-35]}$ Not all of these elevations appear to affect prognosis consistently, especially in non-surgical patients. However, in the perioperative period $\mathrm{cTnT}$ elevation appears to be a robust marker for predicting outcome, and is independently associated with a $10 \%$ 30-day mortality. ${ }^{[5,36]}$ Preoperative hs-cTnT elevation, as well as an absolute rise of $>9 \mathrm{ng} / \mathrm{L} \mathrm{hs}-\mathrm{cTnT}$, have consistently been shown to have a significant short- and long-term effect on perioperative mortality. ${ }^{[37]}$

Our study has implications for cardiovascular risk stratification in SA. Our patients have a similar incidence of MINS to that of international cohorts, despite a possible lower risk profile, which suggests that the current international cardiovascular risk prediction models may be inappropriate for SA patients and/or they may be incorrectly calibrated, resulting in an underestimation of cardiovascular risk in SA surgical patients. This finding is supported by recent literature which suggests that utilising the revised cardiac risk index in SA patients may be inappropriate, especially in vascular surgery ${ }^{[9]}$ Another confounder may be the HIV epidemic in southern Africa, with HIV-positive patients undergoing vascular surgery having fewer of the usually cited cardiovascular risk factors than HIV-negative patients, but similar perioperative morbidity and mortality. ${ }^{[7]}$ The development of an appropriate and well-calibrated perioperative cardiovascular risk prediction model is necessary in SA. A large SA cohort is first necessary to determine calibration of cardiovascular risk factors in SA patients.

The evidence for preventive and therapeutic strategies for MINS is lacking. ${ }^{[18,38]}$ The current literature suggests implementation of simple strategies when MINS is suspected, including appropriate surveillance in the form of regular screening of troponin levels. ${ }^{[18,38]}$ Furthermore, intraoperative prevention should focus on optimising myocardial oxygen supply and demand. Once MINS is diagnosed in the postoperative setting, interdisciplinary management should be initiated. In addition, aspirin and statin therapy show potential benefit, and studies investigating specific therapeutic and preventive measures are in progress. ${ }^{[18]}$

\section{Study limitations}

Although we screened $>1100$ patients, our sample size remained relatively small, and the event rate was lower than expected, so the primary outcome had a wider than anticipated CI. The incidence of MINS in our population was lower than we expected. This may be explained in part by our methodology of single postoperative troponin sampling per patient, while the VISION study had a median of three troponin samples per patient. Furthermore, VISION included both elective and emergency surgery, and as our study only included elective surgeries, this too may have led to a lower incidence of MINS. Together with the lower event rate, our study now appears to be underpowered to achieve all our secondary objectives of establishing associations with MINS. However, as our data suggest a similar incidence of MINS to an international cohort, we would recommend routine perioperative troponin surveillance for intermediate- to high-risk surgical patients in SA.

In addition, the intended multivariate analysis examining the association of patient factors and MINS could not be performed. The high exclusion rate was to avoid confounders for postoperative hs-cTnT elevation that may not have been secondary to myocardial ischaemia. It is possible that the incidence may be higher than reported, since we excluded many patients at high risk of cardiovascular complications who had comorbidities that may be associated with cTnT release due to causes other than ischaemia.

\section{Conclusions}

Since SA still lacks population-specific prognostic values for hs-cTnT, the present study serves as an initial indicator of the incidence of MINS, while also providing guidance for sample size calculation for future larger studies that could identify patient or surgical risk factors for poor outcomes due to MINS. This study failed to demonstrate a difference in the incidence of MINS between our SA cohort and the VISION cohort, although the study limitations weaken our conclusions. Although this study did not specifically examine the association between MINS and major perioperative morbidity and mortality in SA patients, the methodology of the VISION analysis suggests that the associated perioperative morbidity is universally applicable, and our study therefore suggests that MINS has significant implications for public health outcomes in SA. International guidelines promote the utility of troponin surveillance, and a recent study found troponin surveillance to be financially viable in SA. ${ }^{[39,40]}$ Our data suggest that postoperative troponin surveillance is necessary in elevated-risk non-cardiac surgical patients in SA.

Acknowledgements. The authors thank Prof. Bongani Mayosi and his research team for assisting in data collection.

Author contributions. The research protocol was produced by EC, NDM, RAD and BMM. Data collection was done by EC, NDM and CC. The final article was drafted by EC and revised and corrected by BMB and RAD. All authors participated in final proofreading and revision.

Funding. None.

Conflicts of interest. None.

1. Weiser TG, Regenbogen SE, Thompson KD, et al. An estimation of the global volume of surgery: A modelling strategy based on available data. Lancet 2008;372(9633):139-144. https://doi.org/10.1016/ S0140-6736(08)60878-8

2. Boersma E, Kertai MD, Schouten O, et al. Perioperative cardiovascular mortality in noncardiac surgery: Validation of the Lee cardiac risk index. Am J Med 2005;118(10):1134-1141. https://doi. org/10.1016/.jamjmed.2005.01.064

3. Devereaux PJ, Chan MT, Alonso-Coello P, et al. Association between postoperative troponin levels and 30-day mortality among patients undergoing noncardiac surgery. JAMA 2012;307(21):2295-2304. https://doi.org/10.1001/jama.2012.5502

4. Biccard BM, Rodseth RN. The pathophysiology of peri-operative myocardial infarction. Anaesthesia 2010;65(7):733-741. https://doi.org/10.1111/j.1365-2044.2010.06338.x
2010

5. Botto F, Alonso-Coello P, Chan MTV, et al. Myocardial injury after noncardiac surgery. Anesthesiology 2014;120(3):564-578. https://doi.org/10.1097/ALN.0000000000000113

6. Kavsak PA, Walsh M, Srinathan S, et al. High sensitivity troponin $\mathrm{T}$ concentrations in patients undergoing noncardiac surgery: A prospective cohort study. Clin Biochem 2011;44(12):1021-1024. https://doi.org/10.1016/j.clinbiochem.2011.05.017

Redman LA, Naidoo P, Biccard BM. HIV, vascular surgery and cardiovascular outcomes: A South African cohort study. Anaesthesia 2014;69(3):208-213. https://doi.org/10.1111/anae.12521

8. Biccard BM, Naidoo P, de Vasconcellos $\mathrm{K}$. What is the best pre-operative risk stratification tool for major adverse cardiac events following elective vascular surgery? A prospective observational cohort study evaluating pre-operative myocardial ischaemia monitoring and biomarker analysis. Anaesthesia 2012;67(4):389-395. https://doi.org/10.1111/j.1365-2044.2011.07020.x

9. Moodley Y, Naidoo P, Biccard BM. The South African Vascular Surgical Cardiac Risk Index (SAVSCRI): A prospective observational study. S Afr Med J 2013;103(10):746-750. https://doi.org/10.7196/ SAMJ.6967

10. Fleisher L, Fleischmann KE, Auerbach AD, et al. 2014 ACC/AHA guideline on perioperative cardiovascular evaluation and management of patients undergoing noncardiac surgery: A report of the American College of Cardiology/American Heart Association Task Force on Practice Guidelines. he American College of Cardiology/American Heart Association Task Force on
Circulation 2014;130(24):1-105. https://doi.org/10.1161/CIR.0000000000000106

1. Kellum J, Lameire N, Aspelin P, et al. Summary of recommendation statements. Kidney Int Suppl 2012;2(1):8-12. https://doi.org/10.1038/kisup.2012.7

12. KDIGO 2012 Clinical Practice Guideline for the Evaluation and Management of Chronic Kidney Disease: Abstract. Kidney Int Suppl 2013;3(1):1-150. https://doi.org/10.1038/kisup.2012.76 
13. Minitab 17 Statistical Software. 2010. http://www.minitab.com (accessed 25 April 2017).

14. Biccard BM, Madiba TE, Surgical Outcomes Study investigators. The South African Surgical Outcomes Study: A 7-day prospective observational cohort study. S Afr Med J 2015;105(6):465-475. https://doi. org/10.7196/SAMJ.9435

15. Devereaux PJ, Sessler DI. Cardiac complications in patients undergoing major noncardiac surgery. $\mathrm{N}$ Engl J Med 2015;373(23):2258-2269. https://doi.org/10.1056/NEJMra1502824

16. Lee KL, Woodlief LH, Topol EJ, et al. Predictors of 30-day mortality in the era of reperfusion for acute myocardial infarction? Results from an international trial of 41021 patients. Circulation 1995;91(6):1659-1668. https://doi.org/10.1161/01.CIR.91.6.1659

17. Thygesen K, Alpert JS, Jaffe AS, et al. Third universal definition of myocardial infarction. Circulation 2012;126(16):2020-2035. https://doi.org/10.1161/CIR.0b013e31826e1058

18. Mauermann E, Puelacher C, Lurati Buse G. Myocardial injury after noncardiac surgery. Curr Opin Anaesthesiol 2016;29(3):403-412. https://doi.org/10.1097/ACO.0000000000000336

19. Saenger AK, Beyrau R, Braun S, et al. Multicenter analytical evaluation of a high-sensitivity troponin T assay. Clin Chim Acta 2011;412(9-10):748-754. https://doi.org/10.1016/j.cca.2010.12.034

20. Giannitsis E, Kurz K, Hallermayer K, et al. Analytical validation of a high-sensitivity cardiac troponin T assay. Clin Chem 2010;56(2):254-261. https://doi.org/10.1373/clinchem.2009.132654

21. Troponin T hs (high sensitivity) [package insert]. Mannheim, Germany: Roche Diagnostics GmbH, 2013:1-6.

22. Kavsak PA, Allen LC, Apple FS, et al. Cardiac troponin testing in the acute care setting: Ordering, reporting, and high sensitivity assays - an update from the Canadian Society of Clinical Chemist (CSCC). Clin Biochem 2011;44(16):1273-1277. https://doi.org/10.1016/j.clinbiochem.2011.08.00

23. Twerenbold R, Jaffe A, Reichlin T, Reiter M, Mueller C. High-sensitive troponin T measurements: What do we gain and what are the challenges? Eur Heart J 2012;33(5):579-586. https://doi.org/10.1093/ eurheartj/ehr492

24. Henry CR. Myocardial injury and long-term mortality following moderate to severe carbon monoxide poisoning. JAMA 2006;295(4):398-402. https://doi.org/10.1001/jama.295.4.398

25. Frankel WL, Herold DA, Ziegler TW, Fitzgerald RL. Cardiac troponin T is elevated in asymptomatic patients with chronic renal failure. Am J Clin Pathol 1996;106(1):118-123. https://doi.org/10.1093/ ajcp/106.1.118

26. Musso P, Cox I, Vidano E, Zambon D, Panteghini M. Cardiac troponin elevations in chronic renal failure: Prevalence and clinical significance. Clin Biochem 1999;32(2):125-130. https://doi. org $/ 10.1016 / 50009-9120(98) 00089-7$

27. Hafner G, Thome-Kromer B, Schaube J, et al. Cardiac troponins in serum in chronic renal failure. Clin Chem 1994:40(9):1790-1791.

28. Horwich TB. Cardiac troponin I is associated with impaired hemodynamics, progressive left ventricular dysfunction, and increased mortality rates in advanced heart failure. Circulation 2003;108(7):833-838. https://doi.org/10.1161/01.CIR.0000084543.79097.34
29. Bonnefoy E. Serum cardiac troponin I and ST-segment elevation in patients with acute pericarditis. Eur Heart J 2000;21(10):832-836. https://doi.org/10.1053/euhj.1999.1907

30. Baillard C, Boussarsar M, Fosse J-P, et al. Cardiac troponin I in patients with severe exacerbation of chronic obstructive pulmonary disease. Intensive Care Med 2003;29(4):584-589. https://doi. org/10.1007/s00134-003-1635-0

31. Auner HW, Tinchon C, Linkesch W, et al. Prolonged monitoring of troponin $\mathrm{T}$ for the detection of anthracycline cardiotoxicity in adults with hematological malignancies. Ann Hematol 2003;82(4):218222. https://doi.org/10.1007/s00277-003-0615-3

32. Filusch A, Giannitsis E, Katus HA, Meyer FJ. High-sensitive troponin T: A novel biomarke for prognosis and disease severity in patients with pulmonary arterial hypertension. Clin Sc 2010;119(5):207-213. https://doi.org/10.1042/CS20100014

33. Giannitsis E, Müller-Bardorff M, Kurowski V, Weidtmann B. Independent prognostic value of cardiac troponin T in patients with confirmed pulmonary embolism. Circulation 2000;102(2):211-217. https:/ doi.org/10.1161/01.CIR.102.2.211

34. Røsjø H, Varpula M, Hagve T-A, et al. Circulating high sensitivity troponin T in severe sepsis and septic shock: Distribution, associated factors, and relation to outcome. Intensive Care Med 2011:37(1):77-85. https://doi.org/10.1007/s00134-010-2051-x

35. James $\mathrm{P}$, Ellis $\mathrm{CJ}$, Whitlock RM, et al. Relation between troponin $\mathrm{T}$ concentration and mortality in patients presenting with an acute stroke: Observational study. BMJ 2000;320(7248):1502-1504. https:/ patients presenting with an acute stoke
doi.org/10.1136/bmj. 320.7248 .1502

36. Beckman J. Postoperative troponin screening: A cardiac Cassandra? Circulation 2013;127:2253-2256. https://doi.org/10.1161/CIRCULATIONAHA.113.003195

37. Nagele $\mathrm{P}$, Brown $\mathrm{F}$, Gage $\mathrm{BF}$, et al. High-sensitivity cardiac troponin $\mathrm{T}$ in prediction and diagnosis of myocardial infarction and long-term mortality after noncardiac surgery. Am Heart J 2013;166(2):325332. https://doi.org/10.1016/j.ahj.2013.04.018

38. Biccard BM. Detection and management of perioperative myocardial ischemia. Curr Opin Anaesthesiol 2014;27(3):336-343. https://doi.org/10.1097/ACO.0000000000000071

39. Duceppe E, Parlow J, MacDonald P, et al. Canadian Cardiovascular Society guidelines on perioperative cardiac risk assessment and management for patients who undergo noncardiac surgery. Can J Cardio 2017;33(1):17-32. https://doi.org/10.1016/j.cjca.2016.09.008

40. Torborg A, Ryan L, Kantor G, Biccard BM. The pharmacoeconomics of routine postoperative troponin surveillance to prevent and treat myocardial infarction after non-cardiac surgery. S Afr Med J 2014;104(9):619-623. https://doi.org/10.7196/SAMJ.7654

Accepted 8 November 2017 